\title{
MODELACIÓN MATEMÁTICA DEL SISTEMA HIDRÁULICO DE LA RED DE AGUA POTABLE DE LA CIUDAD DE RIOBAMBA
}

\section{Mathematical Modeling of The Hydraulic System of The Potable Water Network of The City of Riobamba}

\author{
${ }^{1}$ Chuquín Nelson*, ${ }^{1}$ Chuquín Daniel, ${ }^{1}$ Miño Gloria, ${ }^{1}$ Chuquín Juan, ${ }^{2}$ Soriano Javier \\ ${ }^{1}$ Grupo de Investigación en seguridad, ambiente y energía. Facultad de Mecánica. \\ Escuela Superior Politécnica de Chimborazo. Riobamba - Ecuador. \\ ${ }^{2}$ Instituto Tecnológico del Agua. Universidad Politécnica de Valencia. \\ Valencia - España. \\ *nelson.chuquin@espoch.edu.ec
}

\begin{abstract}
$\mathbf{R}$
Los sistemas de distribución de agua potable constituyen una pieza clave en el bienestar y el desarrollo de una población, siendo importante el estudio técnico y minucioso de la misma. El objetivo del estudio fue desarrollar un modelo hidráulico de la red de abastecimiento de agua potable de la ciudad de Riobamba, la cual se encuentra distribuido en 9 sectores, misma que suministra el recurso alrededor de 249.891 habitantes.

El modelo desarrollado que simula el comportamiento de la red se realizó en conjunto con la colaboración de la Empresa Municipal de Agua Potable y Alcantarillado de la Ciudad de Riobamba (EMAPAR). Para determinar la eficiencia del modelo se compararon los resultados del modelo desarrollado, con los registrados por la empresa municipal, comprendidos entre los años 2014 y 2015. El estudio en este sentido es de vital importancia, en vista que constituye una herramienta funcional para el análisis dinámico de la red de abastecimiento. De este modo se evaluará puntos estratégicos de la red, donde se evidencia presiones altas en el sistema, mismas que ocasionan fugas en la red disminuyendo su eficiencia volumétrica y aumentando los costos de explotación y distribución del recurso.

El software empleado para la simulación es EPANET (Rosman, 2002) que es una de las herramientas más utilizada para la simulación de redes de abastecimiento de agua, el cual permite al usuario llevar a cabo simulaciones en estado estacionario y en estado dinámico con el fin de evaluar el comportamiento hidráulico del sistema.
\end{abstract}

Palabras claves: EPANET, modelo, red de simulación, simulación.

\section{A bstract}

Distribution systems of drinking water are a key element in the well-being and development of a population, being important the technical and meticulous study of them. The goal of this study was to develop a hydraulic model of the water supply network of the Riobamba City, which is distributed in 9 sectors, and supplies the resource around 249,891 inhabitants. The model developed to simulate the behavior of the network was carried out in conjunction with the collaboration of the municipal water and sewage company of the city of Riobamba (EMAPAR). To determine the Model's efficiency, the results of it, were compared with those of the municipal company between the years 2014 and 2015 . The study 
in this sense is of vital importance, since it constitutes a functional tool for the dynamic analysis of the supply network. In this way, strategic points of the network will be evaluated, where high pressures in the system are evidenced, which cause leaks in the network reducing its volumetric efficiency and increasing the costs of exploitation and distribution of the resource.

The software used for the simulation is EPANET (Rosman, 2002), which is one of the most used tools for the simulation of water supply networks, this allows the user to carry out simulations in steady state and in dynamic state with the to evaluate the hydraulic behavior of the system.

Keywords: EPANET, model, simulation network, simulation.

Fecha de recepción: 03-nov-2017

Fecha de aceptación: 21-may-2018

\section{INTRODUCCIÓN}

Las redes de distribución de agua son infraestructuras hidráulicas que están constituidas por bombas, tanques, reservorios y válvulas. El análisis y modelación a través de modelos hidráulicos en la actualidad están constituyendo una herramienta confiable y eficaz en la toma de decisiones, además de la creación de diferentes escenarios que promuevan en forma conjunta una mejora en el rendimiento, confiabilidad del diseño y eficacia en el suministro de agua potable [1]. Estos modelos son capaces de determinar la velocidad de los flujos, pérdidas unitarias y presiones a lo largo de toda una red para diferentes escenarios de simulación, tales como la variación de los niveles de agua en los depósitos o tanques reservorios, condiciones de las conducciones, esqueletizaciones o ramificaciones de la red, entre otras, y esto lo ejecuta resolviendo interna y simultáneamente ecuaciones de conservación de masa para cada nodo y relacionando la pérdida de carga para cada tubería en la red [2].

El análisis y diseño de redes resulta realmente complejo cuando se tienen redes malladas de abastecimiento como generalmente ocurre en las metrópolis, sin embargo, el comportamiento de la red puede ser descrito por medio de una secuencia de condiciones de estado estacionario, los cuales resultan determinantes para evaluar la adecuación y sistematización de una red. [3]

En los modelos empleados, las ecuaciones hidráulicas son conocidos de tal manera que describen los principales parámetros hidráulicos, tales como: caudal, velocidad y presión de flujo en toda la red de abastecimiento, o ya sea en los nudos de consumo principales, cuyo desempeño será mostrado a través de gráficas tabuladas con objetivos de evaluación [1]. $\quad$ El éxito o fracaso del modelo, respecto a la eficiencia de este, depende directamente de la estimación, de un análisis implícito de sensibilidad de los inputs o parámetros de entrada utili- zados para generar el modelo, así como también de la calibración y validación de este $[4,5]$.

Los modelos de redes de suministro de agua se construyen en función de 2 elementos importantes que son las líneas y los nodos, donde cada línea representa las tuberías del sistema de abastecimiento que estarán interconectadas con los nudos, y cada nudo representa los puntos de consumo de la red [6].

Los nudos de consumo pueden ser de 2 tipos, independientes de la presión (función del volumen) y los que son dependientes de la presión, es decir aquellos donde el suministro de agua depende de la presión hidráulica disponible, por esta razón para obtener resultados más confiables los modelos hidráulicos deben incluir fugas que indudablemente se generan a lo largo de la red de abastecimiento [7].

Las fugas representan pérdidas de agua que es necesario tenerlas en cuenta en la modelación, dado que una red mal gestionada puede reportar valores superiores al 50\% del volumen de entrada de agua al sistema [8-10]. A groso modo, la pérdida de agua en una red de abastecimiento es difícil de cuantificar y localizar, sin embargo, para incluirlas en un modelo hidráulico, generalmente se considera que esta, puede ser distribuida en proporciones iguales en todos los nodos de consumo que componen la red, disminuyendo de esta manera la incertidumbre en la modelación [11]. Además, 
es importante considerar que las características hidráulicas varían espacial y temporalmente en función del patrón de demandas o de la disponibilidad de recurso.

EPANET [12] es una de las herramientas más utilizadas para la simulación de redes de abastecimiento de agua, este permite al usuario llevar a cabo simulaciones tanto en estado estacionario como en estado dinámico con el fin de evaluar el comportamiento hidráulico del sistema y la calidad del agua a lo largo de un período de tiempo en una red de suministro a presión. Este software fue el empleado para el desarrollo del Modelo Hidráulico de la Ciudad de Riobamba.

Área de Estudio

La ciudad de Riobamba está situada a una distancia de $196 \mathrm{Km}$ de Quito (Capital del Ecuador), se encuentra a una altura de $2.750 \mathrm{msnm}$, cuenta con 225.741 habitantes (INEC,2010). El consumo promedio mensual de la localidad se estima en $1.18 \mathrm{Hm} 3$. Por las características de la zona y la disponibilidad del fluido la red de distribución se encuentra sectorizada ( 9 sectores), de tal forma que se mantiene un control por áreas del caudal mensual que demanda y consume cada zona. La particularidad de la red es la presencia de depósitos atmosféricos que distribuyen el agua por gravedad.

\section{MATERIALES Y MÉTODOS}

La metodología para la elaboración del modelo hidráulico sigue el proceso que se ilustra en la Figura.1

\section{Recopilación de datos $y$ Esqueletización de la red}

Los parámetros hidráulicos para la modelación, en lo que respecta a trazado de la red, diámetros, topología y demandas urbanas, se fundamenta en el estudio realizado por Yépez (2014) [13]. Sin embargo, previo a la carga del modelo la base de datos fue depurada a través del

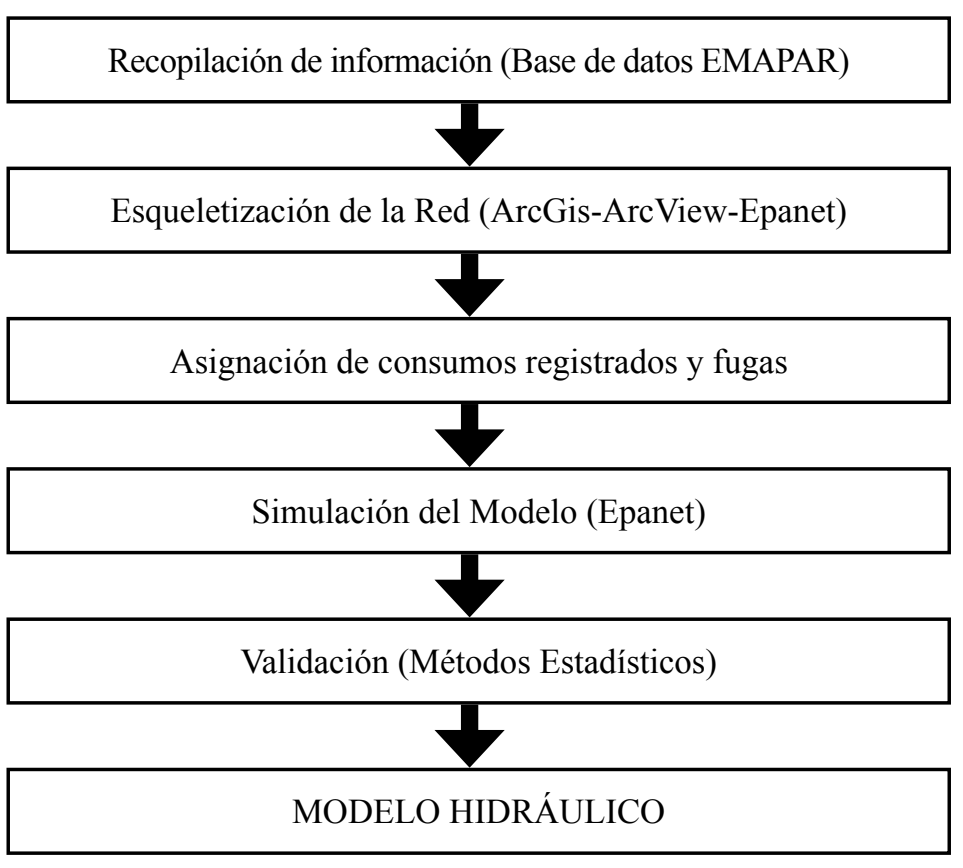

Figura 1. Etapas en la elaboración del Modelo

software ArcView y GisRed para localizar posibles fallos de unión del conjunto tubería - nudo.

\section{Asignación de los consumos y fugas registradas en cada nudo}

Las demandas en los nudos de consumo son fundamentales en las simulaciones, de ellas depende el caudal inyectado y consumido por la red, la fluctuación de nivel de los depósitos, y fundamentalmente la presión disponible en cada nudo. Con el fin de crear un modelo que represente con mayor exactitud las condiciones reales del sistema, se consideraron fugas en el sistema y las mismas fueron analizadas como dependientes de la presión, proponiendo un coeficiente global de fugas y ajustándolo a cada nudo mediante un valor de longitud ponderada de las tuberías [15].

$$
\mathrm{Q}_{\mathrm{F}}=\mathrm{K}_{\mathrm{f}} \sum_{\mathrm{i}=1}^{\mathrm{N}_{\mathrm{j}}} \overline{\mathrm{L}}_{\mathrm{l}}=\left(\mathrm{K}_{\mathrm{i}}\right)^{\alpha}
$$

$$
\mathrm{L}_{1}=\frac{\mathrm{L}_{\mathrm{i}}}{\mathrm{L}_{\mathrm{T}}}
$$


En la Ec.1, QF es el caudal de fugas, Kf es el coeficiente global de fugas, $\mathrm{Nj}$ es el número total de nudos en el modelo, Li es el factor de ponderación de longitud, Pi es la presión en el nudo i, $\alpha$ es el exponente de fugas (para este proyecto se considera el valor de 0.5 ), mientras que en la Ec.2, Li es el $50 \%$ de la longitud de las tuberías conectadas al nudo $\mathrm{i}, \mathrm{L}_{\mathrm{T}}$ es la longitud total de las tuberías del modelo. Los coeficientes emisores (Figura.2) fue determinante para la calibración del modelo y se calculó multiplicando el factor global de fugas por la ponderación de la longitud de las tuberías conectadas a cada nudo en particular.
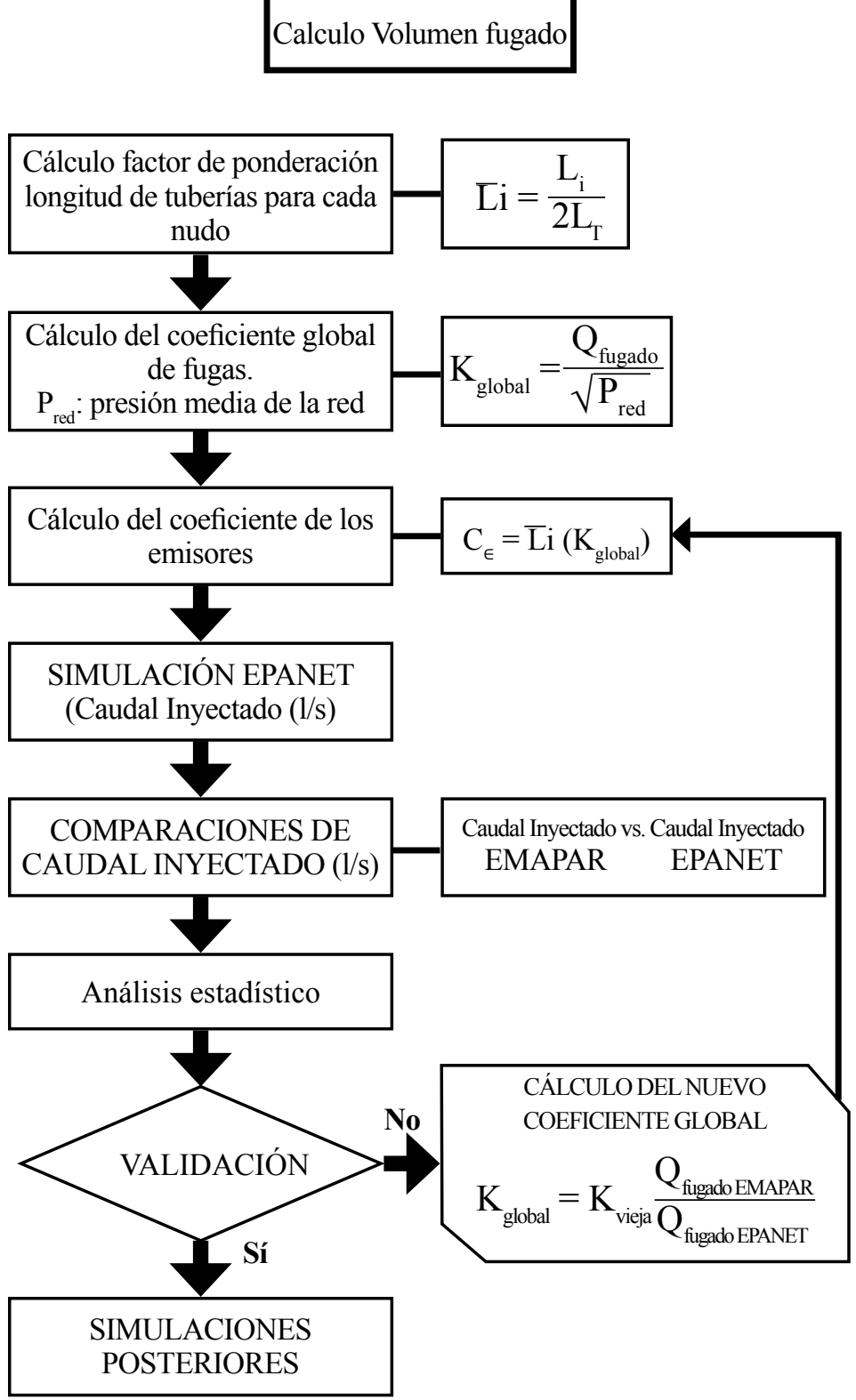

Figura 2. Flujograma para el cálculo de los coeficientes emisores.

\section{Simulación en estado estacionario y dinámico}

La simulación tanto en estado estacionario como dinámico a través del software EPANET, permitió observar la evolución de los caudales, presiones, pérdidas unitarias y velocidades a través de las tuberías y nudos de consumo para cada zona sectorizada del sistema. En referencia a la simulación en estado dinámico, se realizó a escala horaria para un total de 168 horas.

\section{Validación del Modelo}

Los resultados de las simulaciones son válidos siempre y cuando los datos que se obtienen del modelo reproducen con un cierto grado de confianza las características hidráulicas de la red. Para dar el modelo como válido se realizó un análisis de varianza (ANOVA) en 8 puntos estratégicos de la red (1 por cada sector) a intervalos de medición de 12 horas ( $8 \mathrm{H} 00$ - 20H00), durante 7 días donde es posible la monitorización, registro de caudales y velocidades en la red. A partir del ANOVA se evaluó las medias de las variables hidráulicas (presión, velocidad) en cada sector considerando que la presión y velocidad media en cada punto de control es igual a las presiones y velocidades medias de los datos obtenidos del modelo (hipótesis nula).

Además, con el objeto de fortalecer el análisis de varianza y validar la efectividad del modelo hidráulico, se realizó la prueba de rangos múltiples (Test LSD Fisher) para determinar las diferencias de medias posibles, la homogeneidad y valoración de los datos respecto al umbral de velocidades y presiones medias registradas en el sistema. En los dos casos se estableció un intervalo de confianza del 95\%. 


\begin{tabular}{|c|c|c|c|c|c|c|c|}
\hline Sector & $\begin{array}{c}\text { Punto } \\
\text { Monitoreado }\end{array}$ & Fuente & $\begin{array}{c}\text { Suma de } \\
\text { Cuadrados }\end{array}$ & Gl & $\begin{array}{c}\text { Cuadrado } \\
\text { Medio }\end{array}$ & Razón-F & Valor-P \\
\hline \multirow{3}{*}{ Tratamiento } & \multirow{3}{*}{$\mathrm{P} 2$} & Entre grupos & 131.508 & 1 & 131.508 & \multirow{3}{*}{3.08} & \multirow{3}{*}{0.081} \\
\hline & & Intra grupos & 7687.44 & 180 & 42.708 & & \\
\hline & & Total (Corr) & 7818.95 & 181 & & & \\
\hline \multirow{3}{*}{ Tapi } & \multirow{3}{*}{ P4 } & Entre grupos & 2.69218 & 1 & 2.69218 & \multirow{3}{*}{0.21} & \multirow{3}{*}{0.6492} \\
\hline & & Intra grupos & 2334.37 & 180 & 12.9687 & & \\
\hline & & Total (Corr) & 2337.06 & 181 & & & \\
\hline \multirow{3}{*}{ Recreo } & \multirow{3}{*}{ P6 } & Entre grupos & 16.3599 & 1 & 16.3599 & \multirow{3}{*}{2.53} & \multirow{3}{*}{0.1137} \\
\hline & & Intra grupos & 1165.84 & 180 & 6.47687 & & \\
\hline & & Total (Corr) & 1182.2 & 181 & & & \\
\hline \multirow{3}{*}{ Carmen } & \multirow{3}{*}{ P8 } & Entre grupos & 9.61848 & 1 & 9.61848 & \multirow{3}{*}{2.33} & \multirow{3}{*}{0.1288} \\
\hline & & Intra grupos & 743.667 & 180 & 4.13149 & & \\
\hline & & Total (Corr) & 753.286 & 181 & & & \\
\hline \multirow{3}{*}{ Saboya } & \multirow{3}{*}{ P10 } & Entre grupos & 75.7298 & 1 & 75.7298 & \multirow{3}{*}{2.87} & \multirow{3}{*}{0.092} \\
\hline & & Intra grupos & 4750.08 & 180 & 26.3893 & & \\
\hline & & Total (Corr) & 4825.81 & 181 & & & \\
\hline \multirow{3}{*}{ Maldonado } & \multirow{3}{*}{$\mathrm{P} 12$} & Entre grupos & 58.0936 & 1 & 58.0936 & \multirow{3}{*}{3.73} & \multirow{3}{*}{0.0549} \\
\hline & & Intra grupos & 2800.1 & 180 & 15.5561 & & \\
\hline & & Total (Corr) & 2858.19 & 181 & & & \\
\hline \multirow{3}{*}{ Veranillo } & \multirow{3}{*}{ P14 } & Entre grupos & 49.3812 & 1 & 49.3812 & \multirow{3}{*}{3.69} & \multirow{3}{*}{0.0564} \\
\hline & & Intra grupos & 2411.27 & 180 & 13.3959 & & \\
\hline & & Total (Corr) & 2460.65 & 181 & & & \\
\hline \multirow{3}{*}{ Piscin } & \multirow{3}{*}{$\mathrm{P} 16$} & Entre grupos & 10.9227 & 1 & 10.9227 & \multirow{3}{*}{3.13} & \\
\hline & & Intra grupos & 628.381 & 180 & 3.491 & & 0.0786 \\
\hline & & Total (Corr) & 639.303 & 181 & & & \\
\hline & & Entre grupos & 45.5112 & 1 & 45.5112 & & \\
\hline Yaruquíes & P18 & Intra grupos & 2814.31 & 180 & 15.635 & 2.91 & 0.0897 \\
\hline & & Total (Corr) & 2859.82 & 181 & & & \\
\hline
\end{tabular}

Tabla 1. ANOVA (Presión: Registradas - EPANET)

\section{RESULTADOS Y DISCUSIÓN}

\section{Presiones en la Red}

Las mediciones in situ empleadas para la comparación y validación del modelo se realizó en 9 puntos estratégicos de la red en función de la accesibilidad a los puntos de monitorización. En la Tabla 1 se muestra el análisis de varianza, se acepta la hipótesis nula con un intervalo de confianza del 95\%, como se evidencia en cada punto monitoreado (P2, $\mathrm{P} 4$, P6, P8, P10, P12, P14, P16, P18) el valor de $\mathrm{P}$ en todos los casos fue mayor a 0.05 , por tanto, estadísticamente no existe una diferencia significativa entre las medias de los datos monitoreados en la red y lo simulados en EPANET con un nivel de significancia del $5 \%$.

En la Tabla 2 y Figura.3, la prueba de rangos múltiples además corrobora la homogeneidad de los datos comparados, no existe según los resultados del análisis una diferencia significativa entre los conjuntos de datos, el método empleado fue el de diferencia mínima significativa (LSD) con el cual hay un riesgo del $5 \%$ al decir que los pares de datos obtenidos de EPANET son significativamente diferentes a los datos registrados en la red en los puntos de control.

El análisis de presiones deduce que el modelo desarrollado reproduce con un bajo grado de incertidumbre el comportamiento real de la red de suministro. Los resultados de la simulación indican que existen ciertos sectores donde se tienen valores superiores a 50 mca (Nudos Rojos - Figura.4), presiones excesivamente altas, que podrían ocasionar fisuras en la red, lo cual aumentaría 


\begin{tabular}{|c|c|c|c|c|c|c|}
\hline Sector & Punto Registro & Casos & Media (mca) & Grupos Homogéneos & Diferencia & +/- Límites \\
\hline \multirow{2}{*}{ Tratamiento } & P2-EPANET & 91 & 83.9 & $\mathrm{X}$ & \multirow{2}{*}{-1.70008} & \multirow{2}{*}{1.91173} \\
\hline & P2-Observado & 91 & 85.6001 & $\mathrm{X}$ & & \\
\hline \multirow{2}{*}{ Tapi } & P4-EPANET & 91 & 50.1294 & $X$ & \multirow{2}{*}{-0.243246} & \multirow{2}{*}{1.05347} \\
\hline & P4-Observado & 91 & 50.3727 & $X$ & & \\
\hline \multirow{2}{*}{ Recreo } & P6-EPANET & 91 & 36.9003 & $\mathrm{X}$ & \multirow{2}{*}{0.599631} & \multirow{2}{*}{0.744484} \\
\hline & P6-Observado & 91 & 37.4999 & $\mathrm{X}$ & & \\
\hline \multirow{2}{*}{ Carmen } & P8-EPANET & 91 & 19.8 & $\mathrm{X}$ & \multirow{2}{*}{0.459777} & \multirow{2}{*}{0.594602} \\
\hline & P8-Observado & 91 & 20.2598 & $\mathrm{X}$ & & \\
\hline \multirow{2}{*}{ Saboya } & P10-EPANET & 91 & 54.9499 & $\mathrm{X}$ & \multirow{2}{*}{1.29011} & \multirow{2}{*}{1.50275} \\
\hline & P10-Observado & 91 & 56.2401 & $\mathrm{X}$ & & \\
\hline \multirow{2}{*}{ Maldonado } & P12-EPANET & 91 & 51.9501 & $\mathrm{X}$ & \multirow{2}{*}{1.12995} & \multirow{2}{*}{1.15378} \\
\hline & P12-Observado & 91 & 53.08 & $\mathrm{X}$ & & \\
\hline \multirow{2}{*}{ Veranillo } & P14-EPANET & 91 & 35.9818 & $\mathrm{X}$ & \multirow{2}{*}{1.04178} & \multirow{2}{*}{1.07068} \\
\hline & P14-Observado & 91 & 37.0236 & $\mathrm{X}$ & & \\
\hline \multirow{2}{*}{ Piscin } & P16-EPANET & 91 & 25.83 & $\mathrm{X}$ & \multirow{2}{*}{0.489959} & \multirow{2}{*}{0.546573} \\
\hline & P16-Observado & 91 & 26.32 & $\mathrm{X}$ & & \\
\hline \multirow{2}{*}{ Yaruquíes } & P18-EPANET & 91 & 61.5999 & $\mathrm{X}$ & \multirow{2}{*}{1.00012} & \multirow{2}{*}{1.1567} \\
\hline & P18-Observado & 91 & 62.6001 & $\mathrm{X}$ & & \\
\hline
\end{tabular}

Tabla 2. Prueba de Rangos Múltiples (P-Registradas y P-EPANET)
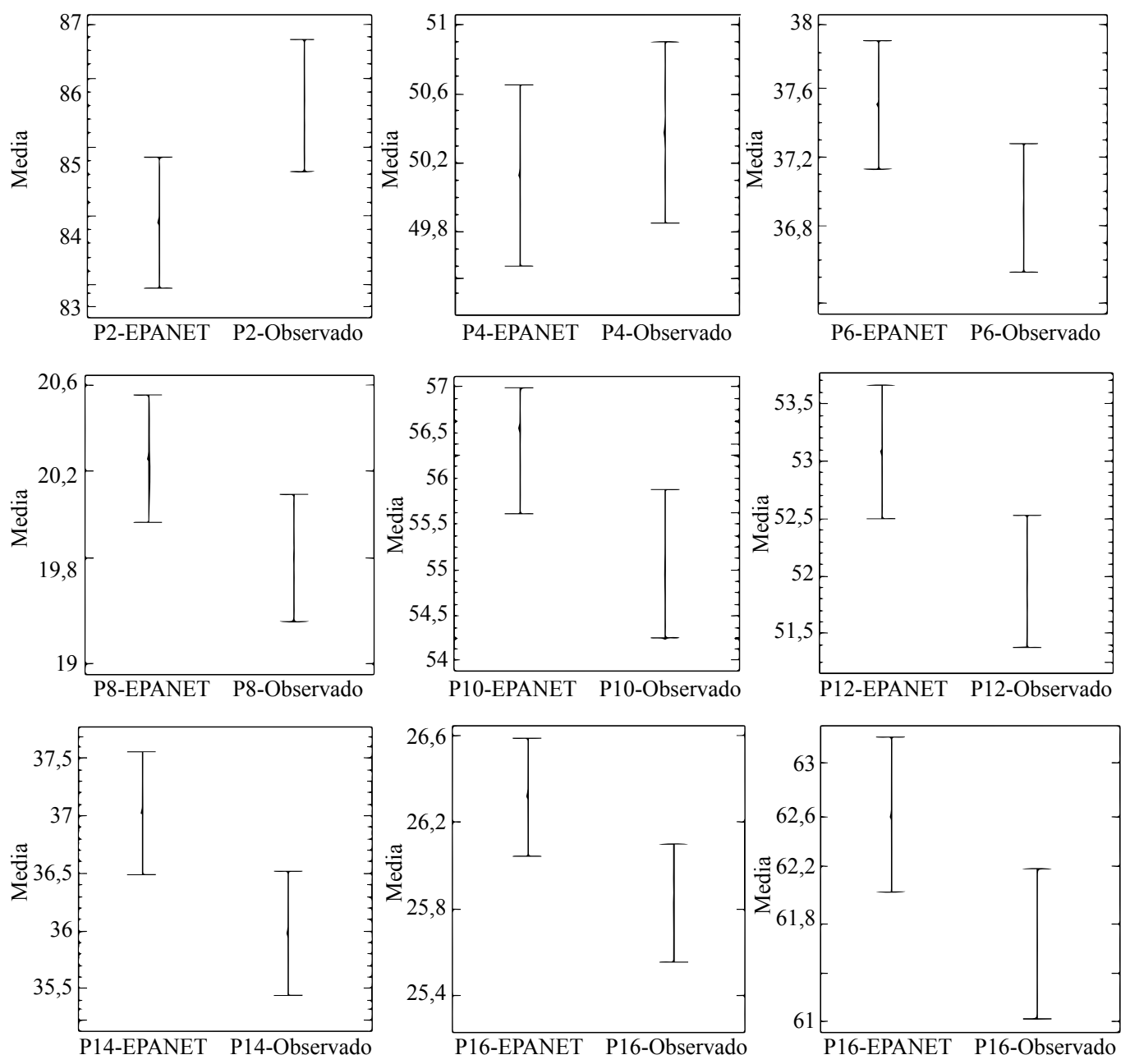

Figura 3. Medias (95\% de Fisher LSD) 


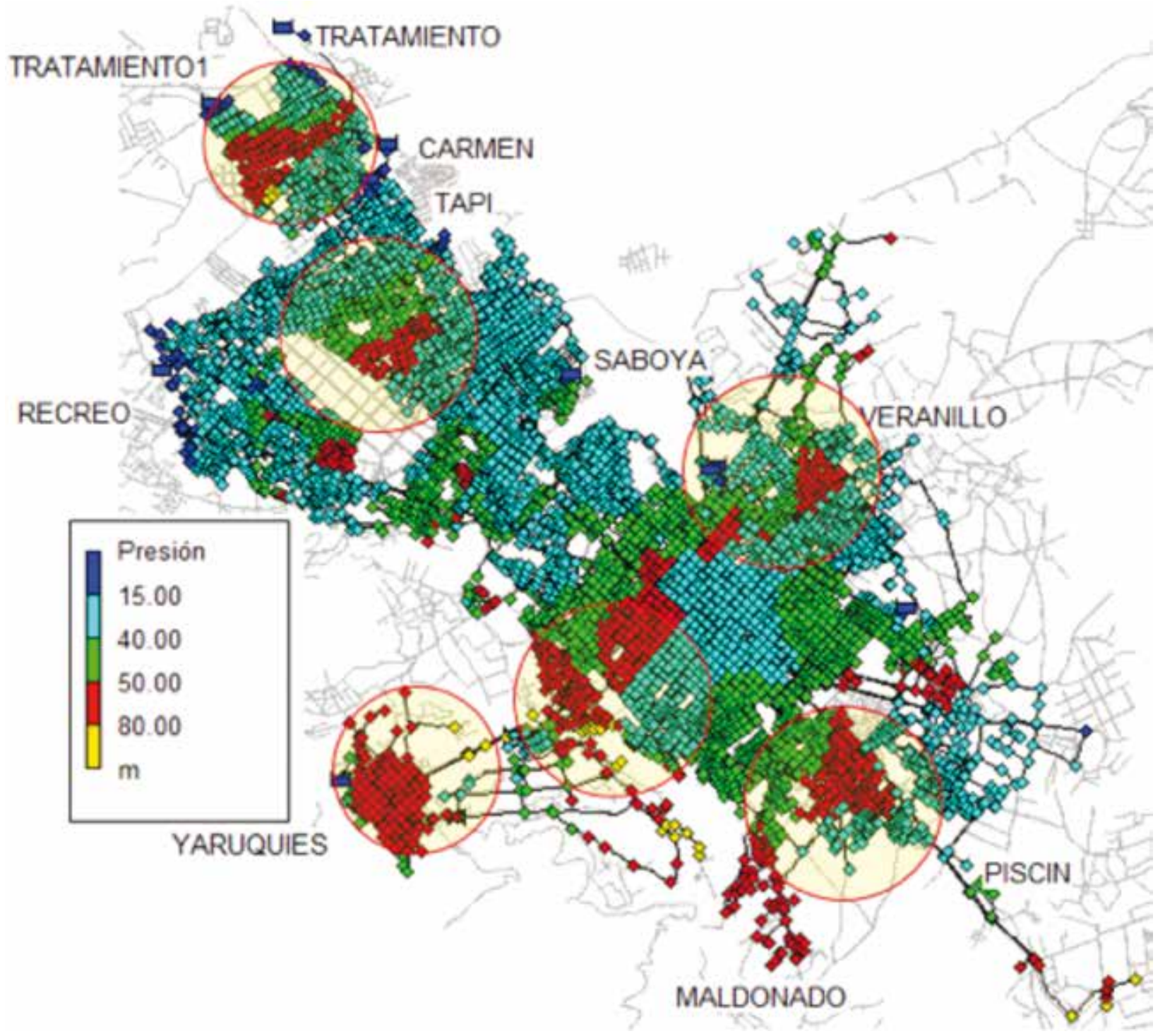

Figura 4. Presiones en la red (Modelo EPANET)

el volumen de agua fugado. Además, el modelo señala que existen puntos donde se tienen presiones del rango de 0 a 15 mca (Nudos Azules - Figura.4), siendo 20 mca la presión recomendada por la AWWA (The American Water Works Association) para evitar posibles intruciones patógenas y la consecuente contaminación del agua [15].

\section{Estudio de velocidades}

La validación de las velocidades del agua en la red se realizó en función de la velocidad media en la tubería principal de cada sector. El análisis de varianza indicó que no existe una diferencia sig- nificativa entre las velocidades medias (valores de $\mathrm{P}>$ 0.05 ) registradas en la red y las obtenidas con el modelo de EPANET (Tabla 3). En la Tabla 4 se especifica la media para cada par de datos y en la misma se detallan intervalos de medida (LSD) bajo las cuales las medias se solaparán el 95\% de las simulaciones realizadas.

La validación del modelo y la simulación extendida posteriori, mostró que el agua en la red adquiere velocidades del rango $(0-3 \mathrm{~m} / \mathrm{s})$, evidenciándose además que la mayor parte de la red en determinadas horas (baja demanda) la velocidad adquiere valores por debajo de 0.10 $\mathrm{m} / \mathrm{s}$ (Líneas Azules - Figura.5), causando efectos contraproducentes en el funcionamiento ideal del sistema, dado que en estas condiciones se favorece la sedimentación de partículas y la acumulación de biofilm en las paredes de las tuberías en el caso que existan averías en la red. Además, estas velocidades bajas promueven el aumento de 


\begin{tabular}{|c|c|c|c|c|c|c|c|}
\hline Sector & $\begin{array}{c}\text { Punto } \\
\text { Monitoreado }\end{array}$ & Fuente & $\begin{array}{l}\text { Suma de } \\
\text { Cuadrados }\end{array}$ & Gl & $\begin{array}{c}\text { Cuadrado } \\
\text { Medio }\end{array}$ & Razón-F & Valor-P \\
\hline \multirow{3}{*}{ Tratamiento } & \multirow{3}{*}{ P2 } & Entre grupos & 0.00309066 & 1 & 0.00309066 & \multirow{3}{*}{0.1} & \multirow{3}{*}{0.7505} \\
\hline & & Intra grupos & 5.48748 & 180 & 0.030486 & & \\
\hline & & Total (Corr) & 5.49057 & 181 & & & \\
\hline \multirow{3}{*}{ Tapi } & \multirow{3}{*}{ P4 } & Entre grupos & 0.00284835 & 1 & 0.00284835 & \multirow{3}{*}{0.08} & \multirow{3}{*}{0.773} \\
\hline & & Intra grupos & 6.14434 & 180 & 0.0341352 & & \\
\hline & & Total (Corr) & 6.14719 & 181 & & & \\
\hline \multirow{3}{*}{ Recreo } & \multirow{3}{*}{ P6 } & Entre grupos & 0.0121984 & 1 & 0.0121984 & \multirow{3}{*}{0.41} & \multirow{3}{*}{0.524} \\
\hline & & Intra grupos & 5.3873 & 180 & 0.0299294 & & \\
\hline & & Total (Corr) & 5.3995 & 181 & & & \\
\hline \multirow{3}{*}{ Carmen } & \multirow{3}{*}{ P8 } & Entre grupos & 0.00184835 & 1 & 0.00184835 & \multirow{3}{*}{0.03} & \multirow{3}{*}{0.8648} \\
\hline & & Intra grupos & 11.4472 & 180 & 0.0635955 & & \\
\hline & & Total (Corr) & 11.449 & 181 & & & \\
\hline \multirow{3}{*}{ Saboya } & \multirow{3}{*}{ P10 } & Entre grupos & 0.00106374 & 1 & 0.00106374 & \multirow{3}{*}{0.01} & \multirow{3}{*}{0.911} \\
\hline & & Intra grupos & 15.275 & 180 & 0.084861 & & \\
\hline & & Total (Corr) & 15.276 & 181 & & & \\
\hline \multirow{3}{*}{ Maldonado } & \multirow{3}{*}{ P12 } & Entre grupos & 0.00184835 & 1 & 0.00184835 & \multirow{3}{*}{0.03} & \multirow{3}{*}{0.8633} \\
\hline & & Intra grupos & 11.1942 & 180 & 0.0621899 & & \\
\hline & & Total (Corr) & 11.196 & 181 & & & \\
\hline \multirow{3}{*}{ Veranillo } & \multirow{3}{*}{ P14 } & Entre grupos & 0.00406374 & 1 & 0.00406374 & \multirow{3}{*}{0.13} & \multirow{3}{*}{0.7159} \\
\hline & & Intra grupos & 5.50665 & 180 & 0.0305925 & & \\
\hline & & Total (Corr) & 5.51071 & 181 & & & \\
\hline \multirow{3}{*}{ Piscin } & \multirow{3}{*}{$\mathrm{P} 16$} & Entre grupos & 0.000494505 & 1 & 0.000494505 & \multirow{3}{*}{0.01} & \\
\hline & & Intra grupos & 6.78294 & 180 & 0.037683 & & 0.9089 \\
\hline & & Total (Corr) & 6.78344 & 181 & & & \\
\hline & & Entre grupos & 0.107829 & 1 & 0.107829 & & \\
\hline Yaruquíes & P18 & Intra grupos & 6.10731 & 180 & 0.0339295 & 3.18 & 0.0763 \\
\hline & & Total (Corr) & 6.21514 & 181 & & & \\
\hline
\end{tabular}

Tabla 3. ANOVA (Velocidades Medias: Registradas - EPANET)

tiempo de residencia del agua en la red, disminuyendo en este sentido la eficiencia de la desinfección (pérdida de la calidad del agua) como consecuencia de la degradación del cloro en la red.

Las velocidades bajas tienen lugar por la existencia de tuberías sobredimensionadas $(400-600 \mathrm{~mm})$, en cuanto a las velocidades máximas (Líneas Amarillas - Figura.5), de acuerdo con el análisis en período extendido se observan valores por encima de $1.5 \mathrm{~m} / \mathrm{s}$ en horas donde $(8 \mathrm{H} 00,13 \mathrm{H} 00,20 \mathrm{H} 00)$ se tienen picos de caudales por las altas demandas, causando en este aspecto importantes pérdidas de carga en forma de energía.

\section{Estudio de pérdidas unitariias}

Este análisis permitió localizar aquellas tuberías que actúan como cuellos de botella disispando energía. Se ha elegido un factor de referencia $(4 \mathrm{mca} /$ $\mathrm{km})$ para determinar si las conducciones generan importantes pérdidas de carga. El análisis en la Figura.6, junto con los resultados que se detallan en la Tabla 5 , muestra que existen sectores (Tratamiento, Tapi, Recreo, Veranillo, Piscin) donde las pérdidas unitarias son despreciables, del orden de 0 - 2mca (Líneas Azules - Figura 6), mientras que en otras (Saboya, Maldonado) se identifica pérdidas superiores a los $4 \mathrm{mca} / \mathrm{km}$ (Líneas Rojas - Figura.6) que deben ser analizadas periódicamente ya que pueden afectar drásticamente las condiciones de funcionamiento y generar fallos indeseables en el sistema de abastecimiento. 


\begin{tabular}{|c|c|c|c|c|c|c|}
\hline \multirow{2}{*}{ Sector } & \multirow{2}{*}{$\begin{array}{l}\text { PUNTOS DE ME- } \\
\text { DICIÓN }\end{array}$} & \multirow{2}{*}{ Casos } & \multirow{2}{*}{ Media $(\mathrm{m} / \mathrm{s})$} & \multicolumn{3}{|c|}{ Error Estándar } \\
\hline & & & & (s agrupada) & Límite inferior & Límite superior \\
\hline \multirow{3}{*}{ Tratamiento } & Vel.P2- EPANET & 91 & 0.427582 & 0.0183033 & 0.402044 & 0.453121 \\
\hline & Vel.P2- Observada & 91 & 0.435824 & 0.0183033 & 0.410286 & 0.461363 \\
\hline & Total & 182 & 0.431703 & & & \\
\hline \multirow{3}{*}{ Tapi } & Vel.P4- EPANET & 91 & 0.77989 & 0.0193678 & 0.752866 & 0.806914 \\
\hline & Vel.P4- Observada & 91 & 0.771978 & 0.0193678 & 0.744954 & 0.799002 \\
\hline & Total & 182 & 0.775934 & & & \\
\hline \multirow{3}{*}{ Recreo } & Vel.P6- EPANET & 91 & 0.548352 & 0.0181355 & 0.523047 & 0.573656 \\
\hline & Vel.P6- Observada & 91 & 0.531978 & 0.0181355 & 0.506674 & 0.557282 \\
\hline & Total & 182 & 0.540165 & & & \\
\hline \multirow{3}{*}{ Carmen } & Vel.P8- EPANET & 91 & 1.20143 & 0.0264358 & 1.16454 & 1.23831 \\
\hline & Vel.P8- Observada & 91 & 1.19505 & 0.0264358 & 1.15817 & 1.23194 \\
\hline & Total & 182 & 1.19824 & & & \\
\hline \multirow{3}{*}{ Saboya } & Vel.P10- EPANET & 91 & 1.46066 & 0.0305375 & 1.41805 & 1.50327 \\
\hline & Vel.P10- Observada & 91 & 1.45582 & 0.0305375 & 1.41322 & 1.49843 \\
\hline & Total & 182 & 1.45824 & & & \\
\hline \multirow{3}{*}{ Maldonado } & Vel.P12- EPANET & 91 & 1.17989 & 0.026142 & 1.14341 & 1.21637 \\
\hline & Vel.P12- Observada & 91 & 1.17352 & 0.026142 & 1.13704 & 1.20999 \\
\hline & Total & 182 & 1.1767 & & & \\
\hline \multirow{3}{*}{ Veranillo } & Vel.P14- EPANET & 91 & 0.487582 & 0.0183352 & 0.461999 & 0.513165 \\
\hline & Vel.P14- Observada & 91 & 0.478132 & 0.0183352 & 0.452549 & 0.503715 \\
\hline & Total & 182 & 0.482857 & & & \\
\hline \multirow{3}{*}{ Piscin } & Vel.P16- EPANET & 91 & 0.75989 & 0.0203494 & 0.731497 & 0.788283 \\
\hline & Vel.P16- Observada & 91 & 0.756593 & 0.0203494 & 0.7282 & 0.784987 \\
\hline & Total & 182 & 0.758242 & & & \\
\hline \multirow{3}{*}{ Yaruquíes } & Vel.P18- EPANET & 91 & 0.795275 & 0.0193094 & 0.768333 & 0.822217 \\
\hline & Vel.P18- Observada & 91 & 0.746593 & 0.0193094 & 0.719651 & 0.773536 \\
\hline & Total & 182 & 0.770934 & & & \\
\hline
\end{tabular}

Tabla 4. Análisis de medias de las velocidades Registradas y Simuladas

\section{Conclusiones}

El presente estudio aplica un modelo hidráulico para el análisis del funcionamiento de la red de abastecimiento de agua potable de la ciudad de Riobamba. A partir del análisis estadístico de varianza, comparación de medias y prueba de rangos múltiples se da como validado el modelo desarrollado, en vista que los principales parámetros hidráulicos (presión, velocidad) reproducen con un intervalo de confianza del 95\% las condiciones bajo las cuales opera el sistema. El análisis en período extendido reveló que existen problemas de gran envergadura en la distribución del fluido. El estudio de las presiones en el sistema evidenció una fluctuación importante de la presión en el rango de 0 a 85 mca, si bien es cierto la presión que garantiza un funcionamiento adecuado es de $20 \mathrm{mca}$, presiones por encima de los 50 mca generan sobrepresiones en el sistema que promueven la generación de fisuras y la consecuente aparición de fugas incontroladas en la red que aumentan el volumen fugado dentro de la instalación, ocasionando pérdidas en rendimiento volumétrico de la red y paralelamente pérdidas económicas importantes por el mal uso del recurso hídrico. Por otro lado, las depresiones que se observan en el sistema son presiones cercanas a 0 mca, lo que puede facilitar la intrusión patógena en el caso que el sistema este próximo a los colectores de aguas residuales, causando un fuerte impacto en la calidad microbiológica del agua, que a posteriori causan la formación de subproductos de cloración (productos cancerígenos), al 


\section{Perfiles
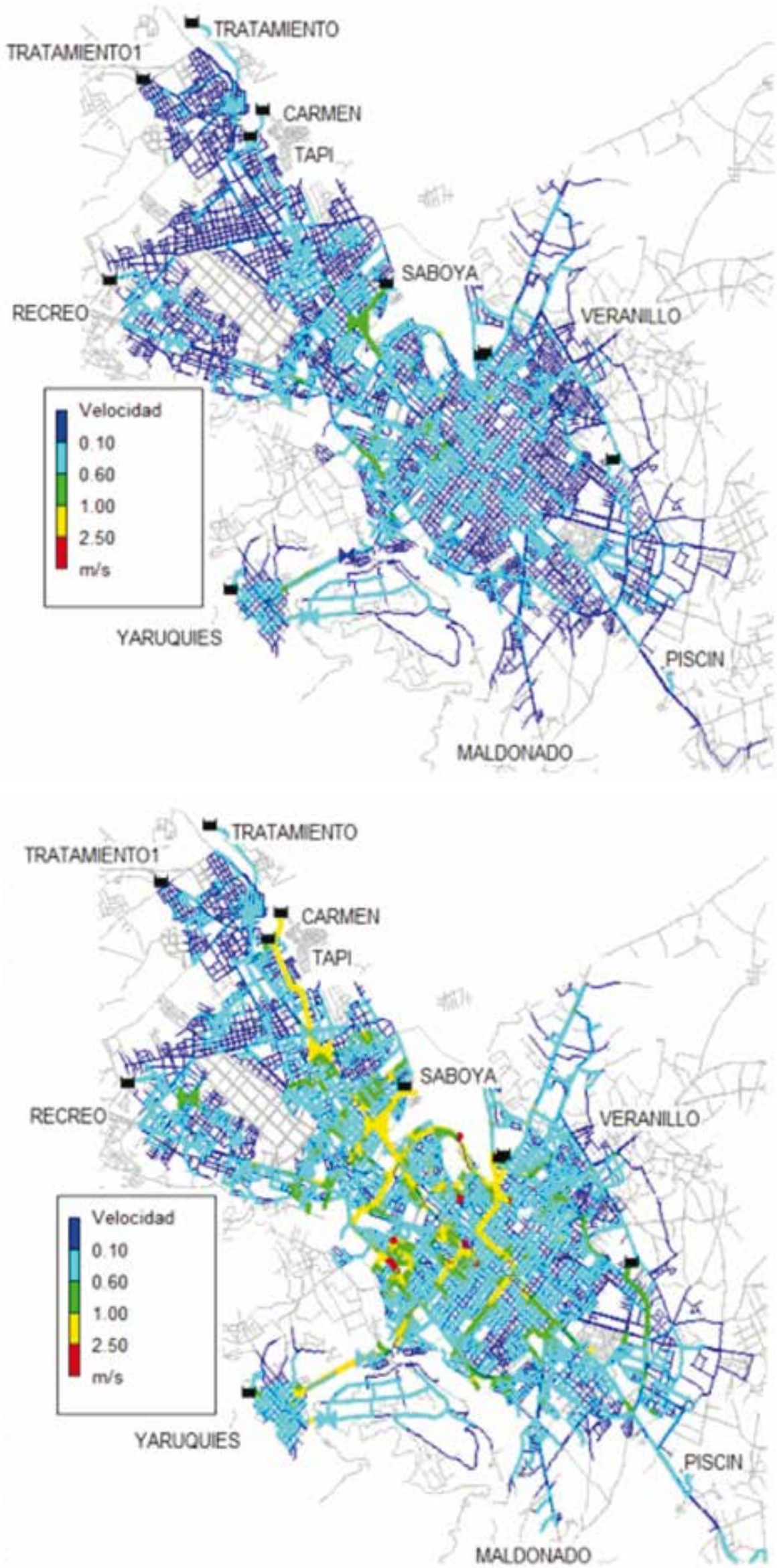


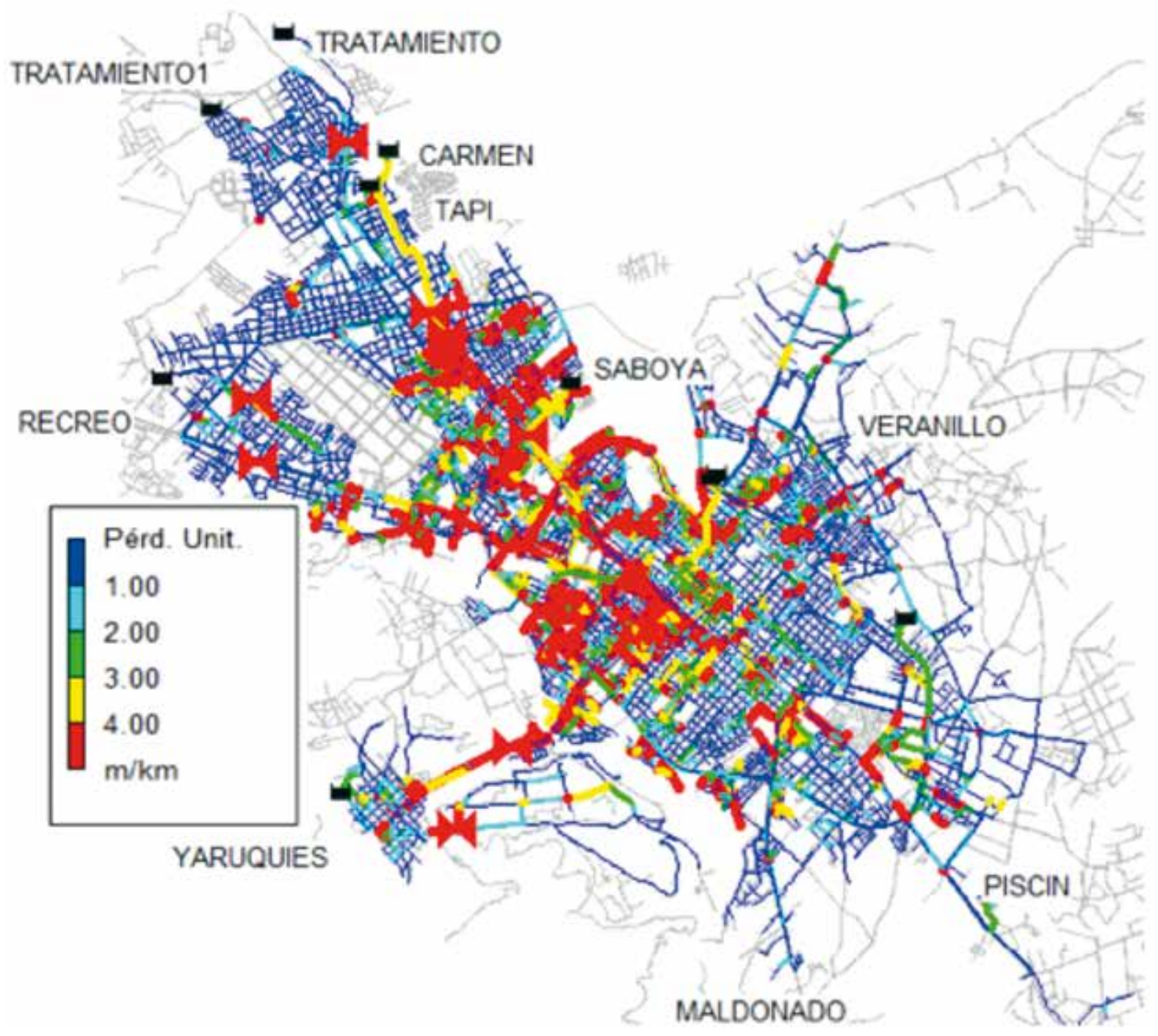

Figura 6. Pérdidas de carga unitarias de las conducciones (mca/km)

\begin{tabular}{|c|c|c|c|c|c|c|c|c|c|}
\hline Hora & Tratamiento & Tapi & Recreo & Carmen & Saboya & Maldonado & Veranillo & Piscin & Yaruquíes \\
\hline $8: 00$ & 0.41 & 1.42 & 0.69 & 1.83 & 5.3 & 4.3 & 0.34 & 1.3 & 1.97 \\
\hline $9: 00$ & 0.45 & 1.58 & 0.75 & 2.15 & 5.4 & 4.6 & 0.39 & 1.49 & 2.19 \\
\hline $10: 00$ & 0.50 & 1.78 & 0.86 & 2.56 & 4.2 & 3.8 & 0.39 & 1.73 & 2.46 \\
\hline $11: 00$ & 0.55 & 2.02 & 0.97 & 3.07 & 3.9 & 3.7 & 0.45 & 2.03 & 2.82 \\
\hline $12: 00$ & 0.59 & 2.2 & 1.06 & 3.43 & 3.85 & 2.9 & 0.51 & 2.24 & 3.06 \\
\hline $13: 00$ & 0.61 & 2.27 & 1.1 & 3.58 & 5 & 5.2 & 0.51 & 2.33 & 3.16 \\
\hline $14: 00$ & 0.57 & 2.09 & 1.01 & 3.2 & 4.8 & 4.9 & 0.51 & 2.11 & 2.91 \\
\hline $15: 00$ & 0.46 & 1.61 & 0.78 & 2.21 & 3.5 & 4.7 & 0.39 & 1.53 & 2.23 \\
\hline $16: 00$ & 0.39 & 1.32 & 0.65 & 1.64 & 3.2 & 3.6 & 0.28 & 1.19 & 1.82 \\
\hline $17: 00$ & 0.36 & 1.19 & 0.56 & 1.39 & 3.6 & 4.1 & 0.28 & 1.03 & 1.64 \\
\hline $18: 00$ & 0.36 & 1.19 & 0.56 & 1.39 & 3.7 & 4.6 & 0.28 & 1.03 & 1.64 \\
\hline $19: 00$ & 0.38 & 1.29 & 0.62 & 1.59 & 4.8 & 5.2 & 0.28 & 1.16 & 1.79 \\
\hline $20: 00$ & 0.42 & 1.46 & 0.71 & 1.92 & 5.2 & 4.9 & 0.34 & 1.35 & 2.02 \\
\hline Promedio & $\mathbf{0 . 4 7}$ & $\mathbf{1 . 6 5}$ & $\mathbf{0 . 7 9}$ & $\mathbf{2 . 3 0}$ & $\mathbf{4 . 3 4}$ & $\mathbf{4 . 3 5}$ & $\mathbf{0 . 3 8}$ & $\mathbf{1 . 5 8}$ & $\mathbf{2 . 2 9}$ \\
\hline
\end{tabular}

Tabla 5. Pérdidas de carga de las conducciones principales de la Red 
reaccionar con el cloro residual de la red si el problema no es controlado.

Además, respecto a las velocidades se ha observado que en varias zonas de la red donde posiblemente existe un sobredimensionamiento de las tuberías, se desarrollan velocidades por debajo de los $0.10 \mathrm{~m} / \mathrm{s}$, lo cual para un sistema de abastecimiento urbano no es lo recomendable, dado que origina paralelamente la sedimentación y adsorción de partículas en las paredes de las tuberías causando progresivamente la disminución del área de flujo y consecuentemente el aumento de las pérdidas totales de carga. Al mismo tiempo, las velocidades bajas estarán asociadas indirectamente a la disminución de la calidad del agua en la red, dado que la degradación del cloro (proveniente de la desinfección) es proporcional al tiempo de residencia del agua en la red, la concentración de cloro puede adoptar valores por debajo de $0.2 \mathrm{mg} / \mathrm{lt}$ (valor recomendado por la Organización Mundial de la Salud para garantizar la inactivación de organismos patógenos causante de enfermedades gastrointestinales), ocasionando a posteriori problemas de salud en los usuarios. Un proyecto de investigación futuro será desarrollar un modelo para evaluar la evolución de cloro en la red y la pérdida de calidad en el sistema tanto en los depósitos de cabecera, como en los depósitos privados instalados por los usuarios.

1. Kara, S., Karadirek, I. E., Muhammetoglu, A., \& Muhammetoglu, H. (2016). Hydraulic Modeling of a Water Distribution Network in a Tourism Area with Highly Varying Characteristics. Procedia Engineering, 162, 521-529.

2. Muranho, J., Ferreira, A., Sousa, J., Gomes, A., \& Marques, A. S. (2014). Pressure-dependent demand and leakage modelling with an EPANET extension-WaterNetGen. Procedia Engineering, 89, 632-639.

3. American Water Works Association, Analysis, and Design of Water Distribution Systems.

4. Sarría, F., \& Palazón, F. (2008). Estimación de parámetros, validación de modelos y análisis de sensibilidad. Modelización de sistemas ambientales. Modelización de sistemas ambientales.

5. Rondán Galán, E. (2016). Estado del arte de la calibración de modelos hidráulicos. Modelado de fugas con Epanet.

6. Caballero, D. (2016). Modelado y simulación de la calidad de agua en una red de abastecimiento municipal: implementación y validación en el municipio de Alcantarilla.

7. Germanopoulos, G. (1985). A technical note on the inclusion of pressure dependent demand and leakage terms in water supply network models. Civil Engineering Systems, 2(3), 171-179.

8. N. Trifunovic, S. Sharma, A. Pathirana, Modelling leakage in distribution system using EPANET, in: Proceedings IWA International Conference WaterLoss2009, Sao Paolo, 2009, pp. 482-489.

9. T. Walski, D. Chase, D. Savic, W. Grayman, S. Beckwith, E. Koelle, Advanced Water Distribution Modelling and Management, first ed., Bentley Institute Pre., London, 2007.

10. V. Kanakouidis, S. Tsitsifli, P. Samaras, A. Zouboulis, Assessing the performance of urban water networks across the EU Mediterranean area: The paradox of high NRW levels and absence of respective reduction measures, Water Sci. Technol. Water Supply 13 (2013) 939-950.

11. A. Bakogiannis, A. Tzamtzis, Modeling of district metered areas with relatively high leakage rate, The case study of Kalipoli's DMA, in: Proceedings of international conference on hydroinformatics, CUNY Academic Works., 2014.

12. ROSSMAN, L. EPANET, Users Manual. Cincinnati: Water Supply and Water Resources Division, United States Environmental Protection Agency, 2002.

13. Yepez, (2014). Sistema de información geográfica (SIG) de predios, acometidas, tuberías y accesorios para la Empresa Pública Empresa Municipal de agua potable y alcantarillado de Riobamba (EP-EMAPAR), Ecuador 
14. Almandoz, J., Cabrera, E., Arregui, F., Cabrera Jr, E., \& Cobacho, R. (2005). Leakage assessment through water distribution network simulation. Journal of water resources planning and management. .15. Mays, L. W. (2000). Water distribution systems handbook. McGraw-Hill. Retrieved from https:// www.accessengineeringlibrary.com/browse/water-distribution-system-handbook 